\title{
Escores Prognósticos de Mortalidade na Cirurgia Cardíaca para Endocardite Infecciosa
}

\author{
Prognostic Scores for Mortality in Cardiac Surgery for Infective Endocarditis
}

\author{
Alexandre Bahia Barreiras Martins ${ }^{1}$ e Cristiane da Cruz Lamas²,3 \\ Unidade Cardiointensiva - Clínica São Vicente da Gávea, ${ }^{1}$ Rio de Janeiro, RJ -Brasil \\ Coordenação de Ensino e Pesquisa - Instituto Nacional de Cardiologia, ${ }^{2}$ Rio de Janeiro, RJ - Brasil \\ Escola de Ciências da Saúde - Universidade do Grande Rio (Unigranrio), ${ }^{3}$ Rio de Janeiro, RJ - Brasil \\ Centro Hospitalar - Instituto Nacional de Infectologia Evandro Chagas - Fiocruz, ${ }^{4}$ Rio de Janeiro, $R J$ - Brasil \\ Minieditorial referente ao artigo: Análise de Escores de Risco para Predição de Mortalidade em Pacientes Submetidos à Cirurgia Cardíaca \\ por Endocardite
}

O artigo de Pivatto F Jr et al., ${ }^{1}$ nos permite discutir a importante questão dos escores prognósticos em pacientes submetidos à cirurgia cardíaca para endocardite infecciosa (EI). ${ }^{1} \mathrm{O}$ manejo da El do lado esquerdo geralmente envolve cirurgia durante a admissão índice, e o principal desafio é identificar rápida e corretamente pacientes de alto risco e transferi-los para instituições com uma equipe cirúrgica com experiência em cirurgia de endocardite.

Os escores prognósticos são importantes por várias razões: uma estimativa razoável do risco de morte é importante na tomada de decisão clínica em relação à indicação cirúrgica; a estimativa é necessária para informar os pacientes e suas famílias sobre o risco cirúrgico; a estratificação de risco permite uma comparação razoável dos resultados da cirurgia cardíaca, para que cirurgiões e hospitais que tratam pacientes de alto risco não pareçam ter piores resultados do que outros. ${ }^{2}$ Para que a mortalidade operatória permaneça uma medida válida da qualidade da assistência, ela deve estar relacionada ao perfil de risco dos pacientes submetidos à cirurgia. ${ }^{2}$

O Euroscore I, publicado em 1999, avaliou 19.030 pacientes submetidos à cirurgia cardíaca em 8 países da Europa, estudando 97 fatores de risco para óbito, dentre os quais foram selecionados aqueles que significativamente afetaram o prognóstico cirúrgico. ${ }^{2}$ Essas variáveis são apresentadas na Tabela 1. Neste estudo, apenas 30\% foram submetidos à cirurgia valvar e o número de indivíduos que apresentou endocardite não é mencionado. ${ }^{2}$

O Euroscore II, publicado em 2012, ${ }^{3}$ teve como objetivo atualizar o primeiro modelo, avaliando 22.381 pacientes de 43 países do mundo, incluindo locais fora da Europa, para criar um escore mais confiável, incorporando novas variáveis e ajustando outras (Tabela 1). Nesta época, já se sabia que o Euroscore2 superestimava o risco cirúrgico, pois progresso

\section{Palavras-chave}

Endocardite/cirurgia; Mortalidade Hospitalar; Cirurgia Cardíaca/mortalidade; Prognóstico; Indicadores.

Correspondência: Cristiane da Cruz Lamas •

Instituto Nacional de Cardiologia - Coordenação de Ensino e Pesquisa - Rua das Laranjeiras, 374 5 andar. CEP 22240-006, Rio de Janeiro, RJ - Brasil Email: cristianelamas@gmail.com

DOI: https://doi.org/10.36660/abc.20200070 técnico em cirurgia cardíaca havia sido alcançado ao longo da década anterior, com uma diminuição da mortalidade ajustada pelo risco. As melhorias no Euroscore foram: clearance de creatinina como uma medida melhor da função renal do que os valores séricos de creatinina; a angina instável definida pelo uso de nitratos intravenosos estava desatualizada; o peso da intervenção não foi avaliado adequadamente no modelo anterior (por exemplo, a troca valvar aórtica com ou sem cirurgia de revascularização do miocárdio concomitante tinha o mesmo peso) e algumas variáveis contínuas, como número de cirurgias cardíacas anteriores e pressões sistólicas da artéria pulmonar, foram tratadas como dicotômicas. ${ }^{3}$

A curva ROC (Receiver Operating Characteristic) dos escores mostrou uma área abaixo da curva (AUC, area under the curve) de 0,78 para o Euroscore logístico e aditivo e de 0,80 para o Euroscore II. Uma crítica ao modelo é que, embora países não europeus tenham sido incluídos, a grande maioria dos pacientes era da Espanha, França, Itália e Reino Unido, que contribuíram com 19, 16, 15 e 12 centros, respectivamente. ${ }^{3}$ Quanto à América Latina, o Brasil contribuiu com dados de 4 centros, Argentina 1 e Uruguai 1. Além disso, o modelo não analisou separadamente a cirurgia valvar. De fato, apenas 2,2\% dos pacientes (497 em números absolutos) com El ativa foram incluídos. ${ }^{4}$ Uma limitação descrita no estudo foi que todos os centros participaram voluntariamente, o que introduz um viés de seleção para os dados. ${ }^{3}$

Pacientes com El precisam ser avaliados minuciosamente. Se tomarmos o perfil habitual de um paciente com El operado no Instituto Nacional de Cardiologia, por exemplo, ele terá uma creatinina sérica acima do normal, com escore de 2 pontos; doença ativa (em tratamento com antibiótico para El no momento da cirurgia), com escore de 3 pontos e disfunção ventricular esquerda pelo menos moderada, com escore de 1 ponto, ou seja, o Euroscore dele seria de 6 pontos e a mortalidade prevista seria superior a 11\%. Não raramente, esse paciente foi submetido a cirurgia cardíaca anterior (já que mais de um terço tem valvopatia reumática e cerca de $10 \%$ tiveram El anterior), o que acrescenta 3 pontos ao escore. ${ }^{4-6}$ Portanto, o Euroscore I não discrimina bem esse subgrupo de pacientes, pois a maioria provavelmente irá apresentar um escore de 6 ou mais. Patrat-Delon et al., ${ }^{7}$ estudando 149 pacientes operados devido a El na França, entre 2002 e 2013, cuja mortalidade hospitalar foi de $21 \%$, chegaram a uma conclusão semelhante em relação ao 
Minieditorial

Tabela 1 - Variáveis incluídas em escores prognósticos de cirurgia cardíaca (Euroscore I e Il e STS-IE)

\begin{tabular}{|c|c|c|c|}
\hline Variáveis & EuroScore I & EuroScore II & STS-IE Score \\
\hline \multicolumn{4}{|l|}{ Idade } \\
\hline \multicolumn{4}{|l|}{ Gênero } \\
\hline \multicolumn{4}{|l|}{ Peso } \\
\hline \multicolumn{4}{|l|}{ Altura } \\
\hline \multicolumn{4}{|l|}{ Diabetes Mellitus } \\
\hline \multicolumn{4}{|l|}{ Doença Pulmonar Crônica } \\
\hline \multicolumn{4}{|l|}{ Arteriopatia Extracardíaca } \\
\hline \multicolumn{4}{|l|}{ Doença Arterial Periférica } \\
\hline \multicolumn{4}{|l|}{ Disfunção Neurológica } \\
\hline \multicolumn{4}{|l|}{ Cirurgia cardíaca prévia } \\
\hline \multicolumn{4}{|l|}{ Número de cirurgias prévias } \\
\hline \multicolumn{4}{|l|}{ Cirurgia valvar prévia } \\
\hline \multicolumn{4}{|c|}{ Insuficiência renal em tratamento conservador } \\
\hline \multicolumn{4}{|l|}{ Insuficiência Renal em Hemodiálise } \\
\hline \multicolumn{4}{|l|}{ Creatinina Sérica/ Clearance de Creatinina } \\
\hline \multicolumn{4}{|l|}{ Arritmia } \\
\hline \multicolumn{4}{|l|}{ Hipertensão Arterial Sistêmica } \\
\hline \multicolumn{4}{|l|}{ Endocardite infecciosa em atividade } \\
\hline \multicolumn{4}{|l|}{ Terapia imunossupressora } \\
\hline \multicolumn{4}{|l|}{ Uso de inotrópicos } \\
\hline \multicolumn{4}{|l|}{ Balão intraaórtico } \\
\hline \multicolumn{4}{|c|}{ Classificação NYHA (New York Heart Association) } \\
\hline \multicolumn{4}{|l|}{ Cirurgia não coronariana } \\
\hline \multicolumn{4}{|l|}{ Angina Instável (CCS IV) } \\
\hline Estado Crítico Pré-operatório & & & \\
\hline Fração de Ejeção do Ventrículo Esquerd & & & \\
\hline Hipertensão Arterial Pulmonar & & & \\
\hline Ressuscitação & & & \\
\hline Procedimento de urgência & & & \\
\hline Peso da Intervenção: & & & \\
\hline Procedimento único não coronariano & & & \\
\hline 2 Procedimentos & & & \\
\hline 3 Procedimentos & & & \\
\hline Ruptura septal pós Infarto do miocárdio & & & \\
\hline
\end{tabular}


EuroSCORE II: subestimou a mortalidade em pacientes com mortalidade prevista acima de $10 \%{ }^{7}$

O escore da Society of Thoracic Surgeons-Infective Endocarditis (STS-IE), publicado em 2011, ${ }^{8}$ tem suas variáveis mostradas esquematicamente na Tabela 1. No subgrupo de pacientes norte-americanos com El estudados, dos 13.617 pacientes, apenas pouco mais da metade apresentava endocardite ativa no momento da cirurgia. ${ }^{8} \mathrm{~A}$ mortalidade geral foi de $8,2 \%$, embora a cirurgia valvar múltipla tivesse uma mortalidade operatória de 13\%. As complicações pós-operatórias estavam presentes em mais da metade dos pacientes, sendo as mais comuns a ventilação prolongada em mais de um quarto.

No escore STS-IE, os números variam de 0 a 110 pontos e, de acordo com esse modelo, um paciente com 35 pontos teria um risco operatório de pelo menos 10\% de mortalidade. ${ }^{8}$ Embora apenas pacientes com El tenham sido estudados, esse foi um registro voluntário exclusivamente de hospitais americanos. Características importantes da El, como microbiologia, discriminação entre válvulas nativas e próteses e presença de complicações intracardíacas (abscesso, fístula) não foram analisadas. Surpreendentemente, 43\% dos pacientes foram operados de maneira "eletiva", o que é um cenário diferente das outras séries.

Embora não sejam específicos para endocardite, o Euroscore e o Euroscore II levam em consideração a endocardite ativa como uma variável importante associada à mortalidade operatória (ver Tabela 1). É importante ressaltar que foram criados vários escores mais específicos para a endocardite, incluindo variáveis com peso significativo em relação à gravidade dessa condição, ${ }^{8-13}$ mostradas na tabela 1 do artigo de Pivatto Jr F et al. ${ }^{1}$ As características específicas da El são: El de válvula protética, grande destruição intracardíaca, Staphylococcus spp., patógeno isolado na cultura de amostras de sangue (ou seja, hemoculturas positivas), presença de abscesso, complicações perivalvares, microrganismo virulento; além destes, há bloqueio atrioventricular e microrganismos Gram-negativos não-HACEK (os últimos 2 para o modelo INC-Rio ${ }^{4}$ ) e envolvimento perivalvar (por exemplo, abscesso anular ou fístula aortocavitária). ${ }^{13}$ Quando agrupados, além do envolvimento da prótese, essencialmente o tipo de microrganismo e a destruição da válvula (bloqueio AV sinalizando abscesso perivalvar) são as características distintivas desses "escores de El" (ver Tabela 2). Mostramos mais dados sobre os escores estudados por Pivatto Jr F et al., ${ }^{1}$ na Tabela 3 e adicionamos a isso os escores INC-Rio ${ }^{4}$ e DeFeo et al. ${ }^{13}$ A mortalidade e a AUC dos escores, em relação à população estudada, são mostradas (Tabela 3). Vale ressaltar que a mortalidade foi variável nas diferentes séries e a mortalidade em pacientes operados com El é pelo menos o dobro da observada em outras cirurgias cardíacas (observe as menores taxas de mortalidade para as populações estudadas no Euroscore I e II). O presente estudo não propõe um escore, e foi adicionado à tabela para mostrar a mortalidade em sua série. Neste estudo ${ }^{1}$, a melhor taxa de mortalidade O/E foi alcançada pelo escore PALSUSE, seguido pelo EuroSCORE logístico, que demonstrou o maior poder discriminatório e foi significativamente superior ao EuroSCORE II, STS-IE, PALSUSE, AEPEI, e RISK-E.

Em conclusão, vários grupos estão em busca de um escore adequado para prever a mortalidade em pacientes operados por El. O Euroscore I e II, amplamente utilizados, e o STSIE, foram estudados comparativamente aos novos escores propostos, alguns dos quais (por exemplo, PALSUSE) incluíram partes do Euroscore. No Brasil, apenas 2 estudos (o presente, com 107 pacientes, e o de Martins et al., ${ }^{4}$ com 154) abordaram o desempenho dos escores na $\mathrm{El}$, ambos com pequeno número. No primeiro, os autores concluíram que, apesar da disponibilidade de escores específicos, o EuroSCORE logístico era o melhor para prever a mortalidade em sua coorte e nenhum escore foi proposto; no segundo, os escores de El mencionados não foram avaliados (a maioria deles publicada após 2016), mas a sensibilidade e especificidade do Euroscore I foram de 81,5\% e 63\%; para Euroscore II, 29,6\% e 97,6\%, e para o STS-IE 7,4\% e 98,4\%, respectivamente. Os valores da AUC foram de 0,86 (Euroscore I), 0,90 (Euroscore II) e 0,85 (STS-IE). Na análise multivariada, as variáveis consideradas estatisticamente significantes para óbito foram bloqueio $\mathrm{AV}$, choque cardiogênico, diabetes mellitus insulinodependente, microrganismos Gram-negativos não-HACEK e uso de inotrópicos. Estes foram incluídos em um modelo, o INC-Rio, ${ }^{4}$ com sensibilidade calculada de $88,9 \%$ e especificidade de 91,8\%; a AUC foi de 0,97. Casalino et al. ${ }^{14}$ estudaram todos os tipos de cirurgia valvar em 440 pacientes, nos quais a taxa de mortalidade foi de 16,0\% (6,0\% em cirurgia eletiva e 34,0\% em cirurgia de emergência / urgência) e constataram que a AUC foi de 0,76 para o EuroSCORE aditivo e logístico e 0,81 para o EuroSCORE II. Eles concluíram que os modelos de EuroSCORE tinham boa capacidade discriminatória, embora a calibração estivesse comprometida devido à subestimação da mortalidade.

Acreditamos que um estudo multinacional no Brasil seria de suma importância, com maior número de pacientes, para propor e validar um escore, uma vez que os pacientes com El em nosso país diferem drasticamente daqueles dos países da América do Norte ou da Europa, principalmente devido à alta proporção de valvopatia reumática, El causada por estreptococos do grupo viridans, maior demora no diagnóstico e idade mais jovem.

\section{Agradecimentos}

Agradecemos ao Dr. Carlos Rochitte, editor-chefe dos Arquivos Brasileiros de Cardiologia, pela oportunidade de debater endocardite infecciosa nesta conceituada revista, e a nossos colegas do Instituto Nacional de Cardiologia pela parceria na "Equipe de Endocardite" e no Mestrado Profissional em Ciências Cardiovasculares.

\section{Fontes de Financiamento}

Fundação de Amparo à Pesquisa do Estado do Rio de Janeiro (FAPERJ; bolsa Jovem Cientista do Nosso Estado,

\# E26/202.782/2015). 
Tabela 2- Variáveis incluídas em escores prognósticos para mortalidade em cirurgia cardíaca em pacientes com endocardite infecciosa submetidos a troca valvar

\begin{tabular}{|c|c|c|c|c|c|}
\hline Variáveis & PALSUSE2014 & AEPEI 2017 & INC-Rio 2016 & $\begin{array}{c}\text { EndoSCORE } \\
2017\end{array}$ & RISK-E 2017 \\
\hline \multicolumn{6}{|c|}{ Endocardite de valva protética } \\
\hline \multicolumn{6}{|l|}{ Idade } \\
\hline \multicolumn{6}{|c|}{ Grande destruição intracardíaca* } \\
\hline \multicolumn{6}{|l|}{ Staphylococcus spp. } \\
\hline \multicolumn{6}{|l|}{ Cirurgia urgente } \\
\hline \multicolumn{6}{|l|}{ Sexo (feminino) } \\
\hline \multicolumn{6}{|l|}{ EuroScore $\geq 10$} \\
\hline \multicolumn{6}{|l|}{$\mathrm{IMC}>27 \mathrm{Kg} / \mathrm{m}^{2}$} \\
\hline \multicolumn{6}{|c|}{ Estado pré-operatório crítico } \\
\hline \multicolumn{6}{|l|}{ Classe IV NYHA } \\
\hline \multicolumn{6}{|l|}{ PSAP > 55mmHg } \\
\hline \multicolumn{6}{|l|}{ DPOC } \\
\hline \multicolumn{6}{|l|}{$\mathrm{Cr} \geq 2 \mathrm{mg} / \mathrm{dL}$} \\
\hline \multicolumn{6}{|l|}{ FEVE } \\
\hline \multicolumn{6}{|c|}{ Número de válvulas/próteses tratadas } \\
\hline \multicolumn{6}{|c|}{ Microrganismo patogênico isolado em hemoculturas } \\
\hline \multicolumn{6}{|c|}{ Presença de abscesso } \\
\hline \multicolumn{6}{|c|}{ Insuficiência renal aguda } \\
\hline \multicolumn{6}{|c|}{ Choque cardiogênico } \\
\hline \multicolumn{6}{|l|}{ Trombocitopenia§ } \\
\hline \multicolumn{6}{|c|}{ Microrganismo virulento"/ } \\
\hline \multicolumn{6}{|l|}{ BAV } \\
\hline \multicolumn{6}{|l|}{ DMID } \\
\hline \multicolumn{6}{|l|}{ GN não-HACEK } \\
\hline Uso de Inotrópicos & & & & & \\
\hline
\end{tabular}

*Abscessos ou outros achados de ecocardiografia sugeriram que a infecção foi invasiva (comunicação entre câmaras, dissecção de parede ou grande deiscência valvular). $¥$ Abscesso, pseudoaneurisma, fistula ou deiscência protética; $\&<150.000$ plaquetas $/ \mathrm{mm}^{3}$. "Staphylococcus aureus ou fungos.

IMC: indice de massa corporal; ClearCreat: clearance de creatinina (taxa de filtração glomerular estimada); NYHA: New York Heart Association; PSAP: pressão sistólica de artéria pulmonar; DPOC: doença pulmonar obstrutiva crônica; Cr: creatinina sérica; FEVE: fração de ejeção do ventrículo esquerdo; BAV: bloqueio atrioventricular; DMID: diabetes mellitus insulinodependente; GN: Gram Negativo; HACEK: Haemophilus spp, Aggregatibacter spp (anteriormente Actinobacillus), Cardiobacterium hominis, Eikenella corrodens, Kingella kingae. 


\section{Minieditorial}

Tabela 3 - Áreas sob a curva (AUC) dos escores de risco propostos para avaliação de mortalidade em cirurgia cardíaca para endocardite infecciosa

\begin{tabular}{lcccccc}
\hline ESCORE & AUC & $\begin{array}{c}\text { Mortalidade } \\
\text { pós- operatória } \\
\text { intra-hospitalar }\end{array}$ & $\begin{array}{c}\text { Avaliou El } \\
\text { separadamente? }\end{array}$ & N estudado & País & Autor, ano \\
\hline Logistic EuroSCORE & 0,79 & $4,7 \%$ & não & 14.799 & Países europeus* & Nashef 1999 \\
\hline EuroSCORE II & 0,81 & $3,9 \%$ & não & 22.381 & $\begin{array}{c}\text { Países } \\
\text { europeus }\end{array}$ & Nashef 2012 \\
\hline STS-IE & 0,76 & $8,2 \%$ & sim & 13.617 & EUA & Gaca 2011 \\
\hline "De Feo"*** & 0,88 & $9,1 \%$ & sim & 440 & Itália & De Feo 2012 \\
\hline PALSUSE & 0,68 & $24,3 \%$ & sim & 437 & Espanha & Martínez-Sellés \\
\hline INC-Rio & 0,97 & $17,5 \%$ & sim & 154 & Brasil & Martins 2016 \\
\hline RISK-E & 0,82 & $28,6 \%$ & sim & 671 & $\begin{array}{c}\text { França e } \\
\text { Espanha }\end{array}$ & Olmos 2017 \\
\hline AEPEI & 0,78 & $15,5 \%$ & sim & 361 & França e Itália & Gatti 2017 \\
\hline EndoSCORE & 0,85 & $11 \%$ & sim & 2.715 & Itália & Di Mauro 2017 \\
\hline Não foi criado & Não avaliada & $29 \%$ & sim & 107 & Brasil & Pivatto Jr F, 2020 \\
\hline
\end{tabular}

AUC: área sob a curva (area under the curve); El: endocardite infecciosa. *Não está discriminado que países participaram. **A maior parte dos centros de investigação eram da França, Itália e Espanha; países da América do Sul e do Norte, Ásia e África tiveram participação pequena. EUA: Estados Unidos da América. ${ }^{* \star *}$ Somente pacientes com El de válvula nativa foram estudados; nenhum nome foi dado ao escore.

\section{Referências}

1. Pivatto Jr F, Bellagamba CCA, Pianca EG, Fernandes FS, Butzke M, Busato SB et al. Analysis of Risk Scores to Predict Mortality in Patients Undergoing Cardiac Surgery for Endocarditis. Arq Bras Cardiol. 2020; 114(3):518-524

2. Roques F, Nashef SA, Michel P, Gauducheau E, de Vincentiis C, Baudet E, et al. Risk factors and outcome in European cardiac surgery: analysis of the EuroSCORE multinational database of 19030 patients. Eur J Cardiothorac Surg. 1999;15(6):816-22.

3. Nashef SA, Roques F, Sharples LD, Nilsson J, Smith C, Goldstone AR, et al. EuroSCORE II. Eur J Cardiothorac Surg. 2012;41(4):734-44.

4. Martins ABB. Avaliação do desempenho de escores de prognóstico de cirurgia cardíaca em pacientes submetidos à troca valvar por endocardite infecciosa no Instituto Nacional de Cardiologia, anos de 2006 a 2016. [dissertação]. Rio de Janeiro: Instituto Nacional de Cardiologia; 2016.

5. Brandão TJ, Januario-da-Silva CA, Correia MG, Zappa M, Abrantes JA, Dantas AM, et al. Histopathology of valves in infective endocarditis, diagnostic criteria and treatment considerations. Infection. 2017;45(2):199-207.

6. Monteiro TS, Correia MG, Golebiovski WF, Barbosa GIF, Weksler C, Lamas CC. Asymptomatic and symptomatic embolic events in infective endocarditis: associated factors and clinical impact. Braz J Infect Dis. $2017 ; 21(3): 240-7$

7. Patrat-Delon S, Rouxel A, Gacouin A, Revest M, Flécher E, Fouquet O, et al EuroSCORE II underestimates mortality after cardiac surgery for infective endocarditis. Eur J Cardiothorac Surg 2016;49(3):944-51.
8. Gaca JG, Sheng S, Daneshmand MA, O'Brien S, Rankin JS, Brennan JM, et al. Outcomes for endocarditis surgery in North America: a simplified risk scoring system. J Thorac Cardiovasc Surg 2011;141(1):98-106.

9. Martínez-Sellés M, Muñoz P, Arnáiz A, Moreno M, Gálvez J, Rodríguez-Roda J, et al. Valve surgery in active infective endocarditis: a simple score to predict in-hospital prognosis. Int J Cardiol. 2014;175(1):133-7.

10. Gatti G, Perrotti A, Obadia J, Duval X, lung B, Alla F, et al. Simple scoring system to predict in hospital mortality after surgery for infective endocarditis. J Am Heart Assoc. 2017;6(7):pii:e004806.

11. Di Mauro M, Dato GMA, Barili F, Gelsomino S, Santè P, Corte AD, et al. Corrigendum to "A predictive model for early mortality after surgical treatment of heart valve or prosthesis infective endocarditis. The EndoSCORE". Int J Cardiol. 2018 May 1;258:337.

12. Olmos C, Vilacosta I, Habib G, Maroto L, Fernández C, López J, et al. Risk score for cardiac surgery in active left-sided infective endocarditis. Heart. 2017;103(18):1435-42.

13. De Feo M, Cotrufo M, Carozza A, De Santo LS, Amendolara F, Giordano S, et al. The need for a specific risk prediction system in native valve infective endocarditis surgery. ScientificWorldJournal. 2012;2012:307571.

14. Casalino R, Tarasoutchi F, Spina G, Katz M, Bacelar A, Sampaio R, et al. EuroSCORE models in a cohort of patients with valvular heart disease and a high prevalence of rheumatic fever submitted to surgical procedures. PLoS One. 2015;10(2):e0118357. 